\title{
ANTIPLATELET THERAPY The GRAVITAS of clopidogrel dose
}

High-dose clopidogrel treatment in patients who underwent percutaneous coronary intervention (PCl) and have high on-treatment platelet reactivity is associated with similar efficacy and safety to use of a standard dose of the drug, show findings from the GRAVITAS trial published in JAMA.

Combination therapy with aspirin and a $\mathrm{P}_{2} \mathrm{Y}_{12}$ antagonist, such as clopidogrel, is recommended for patients undergoing PCl. However, the level of platelet inhibition achieved with clopidogrel treatment varies considerably across patients. Some studies have suggested that high on-treatment platelet reactivity when taking clopidogrel (that is, a diminished effect of the drug) is associated with increased risk of major adverse cardiovascular events after PCl. "Whether the clinician could alter antiplatelet treatment and improve patient outcomes based on measuring the level of platelet reactivity was unknown," explains Matthew Price, the lead investigator of GRAVITAS. "Therefore, we sought, in the setting of a randomized clinical trial, to test the safety and efficacy of a high-dose strategy in patients with high on-treatment [platelet] reactivity after PCI."

The GRAVITAS trial was conducted in 83 centers in the USA and Canada. The study involved 5,429 patients who received one or more drug-eluting stents for treatment of stable coronary artery disease, non ST-segment elevation acute coronary syndromes, or ST-segment elevation myocardial infarction between July 2008 and April 2010. The GRAVITAS investigators assessed platelet reactivity in these patients with the VerifyNow $\mathrm{P}_{2} \mathrm{Y}_{12}$ test (Accumetrics, San Diego, CA, USA) $12-24 \mathrm{~h}$ after PCI. Patients were only enrolled if they had received periprocedural clopidogrel treatment that made certain that they were at or near a steady state of platelet inhibition at the time of testing. Patients with high on-treatment platelet reactivity $(n=2,214)$ were randomly assigned to receive a $600 \mathrm{mg}$ dose of clopidogrel on the first day of treatment followed by a $150 \mathrm{mg}$ daily dose of the drug ( $n=1,109)$, or to receive a $75 \mathrm{mg}$ daily dose of clopidogrel from the beginning of the study $(n=1,105)$.

After 6 months, the composite (primary) end point of death from cardiovascular causes, nonfatal myocardial infarction, or stent thrombosis, was not different in the high-dose and standard-dose groups of patients with high platelet reactivity (2.3\% vs $2.3 \%$; hazard ratio $[\mathrm{HR}] 1.01 ; 95 \% \mathrm{Cl} 0.58-1.76 ; P=0.97)$, and a similarity was also observed at 30 days (1.9\% vs $1.6 \%$; HR 1.19 ; 95\% $\mathrm{Cl} 0.62-2.27$; $P=0.60$ ). The 6-month rate of severe or moderate bleeding as defined according to GUSTO criteria (safety end point) was also similar between the two groups (1.4\% vs 2.3\%; HR 0.59; 95\% Cl 0.31-1.11; $P=0.10$ ).

High-dose clopidogrel treatment had only a modest pharmacodynamic effect in this group of patients. "This begs the question of whether these patients were, in a sense, undertreated by the intervention we tested," says Price. "We are currently analyzing the GRAVITAS results to see whether a specific achieved level of platelet reactivity was associated with a lower event rate," he adds. The researchers are also investigating whether the presence of specific genetic polymorphisms was associated with a lack of response to treatment with the high clopidogrel dose.

Joana Osório

Original article Price, M. J. et al. Standard- vs high-dose clopidogrel based on platelet function testing after percutaneous coronary intervention: the GRAVITAS randomized trial. JAMA 305, 1097-1105 (2011) 